\title{
Translation by Ribosomes with mRNA Degradation: Exclusion Processes on Aging Tracks
}

\author{
Apoorva Nagar · Angelo Valleriani · Reinhard Lipowsky
}

Published online: 21 September 2011

(C) The Author(s) 2011. This article is published with open access at Springerlink.com

\begin{abstract}
We investigate the role of degradation of mRNA on protein synthesis using the totally asymmetric simple exclusion process (TASEP) as the underlying model for ribosome dynamics. mRNA degradation has a strong effect on the lifetime distribution of the mRNA, which in turn affects polysome statistics such as the number of ribosomes present on an mRNA strand of a given size. An average over mRNA of all ages is equivalent to an average over possible configurations of the corresponding TASEP — both before steady state and in steady state. To evaluate the relevant quantities for the translation problem, we first study the approach towards steady state of the TASEP, starting with an empty lattice representing an unloaded mRNA. When approaching the high density phase, the system shows two distinct phases with the entry and exit boundaries taking control of the density at their respective ends in the second phase. The approach towards the maximal current phase exhibits the surprising property that the ribosome entry flux can exceed the maximum possible steady state value. In all phases, the averaging over the mRNA age distribution shows a decrease in the average ribosome density profile as a function of distance from the entry boundary. For entry/exit parameters corresponding to the high density phase of TASEP, the average ribosome density profile also has a maximum near the exit end.
\end{abstract}

Keywords mRNA translation · Degradation · TASEP

A. Nagar $(\bowtie) \cdot$ A. Valleriani $\cdot$ R. Lipowsky

Department of Theory and Bio-Systems, Max Planck Institute of Colloids and Interfaces, 14424

Potsdam, Germany

e-mail: apoorva.nagar@mpikg.mpg.de

A. Valleriani

e-mail: Angelo.Valleriani@mpikg.mpg.de

R. Lipowsky

e-mail: Reinhard.Lipowsky@mpikg.mpg.de

Present address:

A. Nagar

Department of Physics, Indian Institute of Technology Hyderabad, Yeddumailaram, Andhra Pradesh 502205, India 


\section{Introduction}

Translation is an essential step during protein synthesis, in which ribosomes move on mRNA strands to produce proteins. Understanding protein production is a problem of central importance in biology and, therefore, translation has attracted considerable experimental effort. The theoretical challenge is to identify important mechanisms and develop simple models that capture the experimental results and have predictive power. The totally asymmetric simple exclusion process (TASEP) is a nonequilibrium lattice model that has been intensely studied in connection to mRNA translation [1-5]. TASEPs are interesting models in themselves and have emerged as a paradigm in nonequilibrium physics because they lead to nontrivial but analytically tractable behavior $[6,7]$.

The translation process consists of three steps: initiation, elongation and termination. During initiation, two ribosomal subunits dispersed in the cytosol are assembled at the start codon of the mRNA. The ribosomes now move forward, assembling the protein by attaching one amino acid at a time. These amino acids, carried by tRNA, arrive randomly and the one matching the codon at the ribosome location is accepted and added to the protein chain. Thus, the movement of the ribosomes is unidirectional but stochastic. Once a ribosome reaches the end codon, it disassembles and the protein is released. It is easy to see that the TASEP with open boundaries is a suitable model for translation since it describes particles that obey hard core exclusion, move stochastically in a single direction, enter the lattice at one end and leave it at the other end with certain rates, which determine the steady state phases of the system. The typical approach to model mRNA translation with TASEP was, until now, to consider a steady state condition in which the number of ribosomes on the mRNA had reached its stationary distribution. A more realistic view of translation, however, should incorporate the effect of degradation of mRNA, which makes it important to consider the effects of the transient state before the stationary distribution has been reached.

In this paper, we use TASEPs to study the effect of degradation of mRNA on translation. The degradation of mRNA is caused by RNA-degrading proteins [8] and can occur at any stage of the mRNA life cycle [8-12]. In prokaryotic cells the mRNA can be degraded cotranslationally, all ribosomes that are on the mRNA at the moment of degradation are lost and the mRNA chains are degraded immediately $[9,11,13]$. In eukaryotic cells, the degradation of mRNA proceeds in two steps. First, the initiation region is decapped to prevent the assembly of new ribosomes. Second, the ribosomes present on the mRNA at the moment of decapping are allowed to finish their translation $[9,14]$. While degradation of mRNA before completion of protein production looks like a wasteful process at first sight, it is also a control mechanism that enhances the efficiency of protein synthesis [15]. The effects of mRNA degradation have largely been overlooked in previous studies of dynamical models. Usually, it is assumed that a steady state average should reflect the experimental observations $[2,3,5]$. In reality, the cell extract is expected to contain mRNA of various ages, all of which will contribute to the average, see Fig. 1. We have tried to capture this effect in our recent work $[16,17]$ where we have studied a simple model with very low entry rates, neglecting the hard core exclusion interaction between the ribosomes. We found that degradation can have a significant effect on experimentally measurable quantities such as the polysome size distribution and the density of ribosomes as a function of codon position. We also showed that degradation induces a length dependence in the translation rate leading to a decrease in the steady state amount of proteins per mRNA, the so-called translational ratio, with increasing protein length [17].

Since exclusion effects can significantly reduce the efficiency of mRNA translation, mRNA degradation could be a mechanism that helps avoiding major efficiency loss. Indeed, 


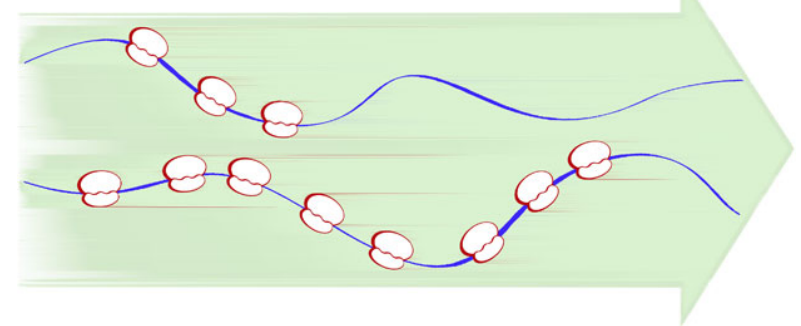

Fig. 1 Schematic diagram of mRNA translation for different ages. The two chains have the same length $L$ but differ in the number of loaded ribosomes. The upper chain is young and carries only a few ribosomes that are close to the initiation region on the left. The chain at the bottom is older and thus is loaded with more ribosomes. The ribosomes move from left to right as indicated by the large background arrow

degradation favors younger, sparsely loaded mRNA thus reducing the effect of ribosome exclusion. It is therefore important to include both exclusion effects and degradation in the theoretical description. In the present work, we consider the exclusion interaction between ribosomes, which was neglected in our earlier work in [16]. We use TASEPs to describe the ribosome dynamics, and the quantities of interest are calculated by averaging over the mRNA age distribution. This involves taking an average over system configurations both before and after steady state has been attained. While the steady state properties of TASEPs have been very well studied, the approach to steady state is a more difficult problem with fewer analytical results $[18,19]$.

In this paper, we use discrete time stochastic simulations and mean field approximations to study the approach towards the steady states of TASEPs when the initial state corresponds to completely empty mRNA strands. We numerically evaluate the total number of ribosomes on the mRNA as a function of time and then use mean field arguments to understand these results. We show that these arguments work fairly well for the high and low density phases. A slight discrepancy is found in the maximal current phase. We then use these results to calculate the quantities of interest for the translation problem by taking an average over the age distribution.

Our results show that the average ribosome density profile can decrease significantly as we move along the mRNA. This decrease is caused by averaging over mRNA of various ages-younger mRNA strands, for which steady state has not been reached, contribute more to the density of the initial part of the coding region. This leads to another interesting consequence - the average density of ribosomes decreases as a function of the length of the mRNA coding region. New experimental methods [20-24] allow for the measurements of these quantities, and we expect that a detailed comparison between theory and experiment will soon be possible.

The paper is organized as follows: In Sect. 2, we explain our theoretical approach. We then describe the results for the approach to steady state of the TASEP in Sect. 3. Section 4 deals with the results for the mRNA translation. Here we report our computational results for several quantities of interest, and compare them with approximations developed using our results in Sect. 3. We discuss the implications of our theoretical results for experiments in Sect. 5. To focus on translation, all our discussion below will be in terms of "ribosomes" and "mRNA" instead of the usual terminology of "particles" and "lattice". It is straightforward, however, to rephrase our results in the usual TASEP terminology and to apply it to different contexts. 


\section{Theoretical Description}

A single mRNA strand is represented by a one dimensional lattice with $L$ sites, which corresponds to the coding region. Ribosomes-which are equivalent to particles interacting with each other via hard core repulsion-enter the mRNA at the initiation lattice site $z=1$ with rate $\omega_{\text {on }}$, walk in a directed fashion from site to site with a rate $t_{0}^{-1}$ until they reach the last lattice site $z=L$, and leave the mRNA from the termination lattice site $z=L$ with rate $\omega_{\mathrm{ex}}$. The basic time scale $t_{0}$ in the system is determined by the ribosome dynamics. The distance between two mRNA sites is taken to be $l_{0}$ which is the longitudinal extension of a ribosome in codons and each ribosome attempts a forward move in time $t_{0}$, implying that an unhindered ribosome will move with a velocity $v_{0}=l_{0} / t_{0}$ codons per time unit or $v_{0}=t_{0}^{-1}$ footprints per time unit. Since $t_{0}$ is the basic unit of time, $\omega_{\mathrm{on}}$ and $\omega_{\mathrm{ex}}$ are related to the entry and exit rates in the usual TASEP notation by $\alpha=\min \left(\omega_{\mathrm{on}} t_{0}, 1\right)$ and $\beta=\min \left(\omega_{\mathrm{ex}} t_{0}, 1\right)$. The lattice parameter $l_{0}$ is of the order of 10 codons and, thus, implies a certain coarse graining of the ribosome dynamics since displacements (or translocations) of the ribosomes corresponding to single codons are not resolved.

Since the binding processes of the transfer RNA molecules to the different ribosomes represent statistically independent events, the new ribosome configuration after the next time step is determined by a random, asynchronous update, i.e. by choosing a ribosome at random and attempting to move it forward to the next binding site at the mRNA. One time step consists of $M(t)$ such moves where $M(t)$ is the total number of ribosomes on the mRNA at time $t$. This process corresponds to the usual TASEP.

The random life time $U$ of the mRNA is described by a turnover time distribution, which, for a fixed degradation rate $\omega_{\mathrm{de}}$, is given by $\phi_{U}(t)=\omega_{\mathrm{de}} \exp \left(-\omega_{\mathrm{de}} t\right)$. In general, mRNA degradation is balanced by mRNA production via continuous transcription. We will assume that this balance leads to a time-independent average number of mRNA molecules within the cytosol. The mRNA age distribution is then given by

$$
\phi_{A}(t)=\omega_{\mathrm{de}} \exp \left(-\omega_{\mathrm{de}} t\right)
$$

as was shown in [16]. At time $t=0$, the mRNA molecules carry no ribosomes, which start loading at one end of the mRNA and moving towards the other end as the age of the mRNA increases. The relevant quantities will be calculated by taking an average over all ages of mRNA. While the actual age distribution of mRNA age may not always be exponential, the qualitative features of our results are expected to apply to other age distributions as well.

For the sake of clarity, we shall give here the precise definitions of the quantities that we are going to study in the next section and, thus, also fix our notation. The random number of ribosomes on an mRNA of age $t$ will be distributed according to the probability distribution $P_{n}(t)$, for any integer $0 \leq n \leq L$, where $L$ is the length of the mRNA expressed in footprints. $L$ therefore gives the maximal number of ribosomes that can be loaded on an mRNA which has $L l_{0}$ codons in the coding region. Thus, $P_{n}(t)$ gives the probability that an mRNA of age $t$ is loaded with exactly $n$ ribosomes. When there is no degradation, $P_{n}(t)$ will converge to the usual TASEP steady state distribution $P_{n}^{\text {st }}$ at large times. Incorporating the effect of degradation involves weighting the distribution $P_{n}(t)$ with the age distribution $\phi_{A}$ which leads to the average probability distribution

$$
\Pi_{n}=\left\langle P_{n}\right\rangle \equiv \int_{0}^{\infty} \mathrm{d} t P_{n}(t) \phi_{A}(t)
$$

where $\phi_{A}(t)$ is defined in (1). Notice that henceforth we will use the word average and the symbol $\langle\cdot\rangle$ to indicate averages over the age distribution $\phi_{A}$. 
The quantity $M(t)$ already introduced before is given by

$$
M(t)=\sum_{n=0}^{L} n P_{n}(t)
$$

as the mean over the distribution $P_{n}(t)$ and represents the average number of ribosomes on an mRNA of age $t$. Again, in the case of no degradation and large $t$, the number of ribosomes will converge to the TASEP stationary value $M^{\text {st }}$, whose value will depend on the choices of the rates $\omega_{\mathrm{on}}$ and $\omega_{\mathrm{ex}}$, as we shall see. The convolution of $M(t)$ with the age distribution $\phi_{A}$ as given by

$$
\langle M\rangle=\int_{0}^{\infty} \mathrm{d} t M(t) \phi_{A}(t),
$$

represents the average number of ribosomes on a randomly chosen chain. The quantity, $\langle M\rangle / L$ will be called the average ribosome density.

Finally, we will denote by $m(z, t)$ the density profile of ribosomes at position $z=$ $1,2, \ldots, L$ along an mRNA of age $t$. This profile represents the probability to find a ribosome at position $z$ on a mRNA of age $t$. For large $t$, the density profile will become stationary and we have $m(z, t) \approx m^{\text {st }}(z)$. As previously, $m^{\text {st }}(z)$ represents the steady state value without degradation, whereas degradation leads to

$$
\langle m(z)\rangle=\int_{0}^{\infty} \mathrm{d} t m(z, t) \phi_{A}(t),
$$

which gives the profile averaged over chains of all ages.

The quantities that are experimentally accessible are the averaged quantities $\Pi_{n}$ and $\langle M\rangle$ from centrifugation techniques, and $\langle m(z)\rangle$ from footprinting (deep sequencing) techniques. If the typical life time of the mRNA is much larger than the transient times to reach steady state, the age-averaged quantities in $(2,4,5)$ will not differ much from their usual stationary counterparts. Thus, in a general biological context, deviations of the averaged quantities $\Pi_{n},\langle M\rangle$ and $\langle m(z)\rangle$ from the stationary quantities $P_{n}^{\text {st }}, M^{\text {st }}$ and $m^{\text {st }}(z)$, respectively, should reflect the rapid turn-over of the mRNA.

\section{TASEP Evolving to Steady State}

In this section, we will describe our results on the time evolution of a TASEP. We consider an mRNA that is initially unoccupied and evolves towards steady state with time. We are interested in studying the density profile and the total number of ribosomes on the mRNA as a function of time.

Before describing our results for the approach to the steady state, we will summarize some known results for this steady state. The phase diagram is displayed in Fig. 2. The diagram shows three distinct phases depending on the entry and exit rates, $\omega_{\mathrm{on}}$ and $\omega_{\mathrm{ex}}$ respectively.

For $\omega_{\mathrm{on}} t_{0}<\omega_{\mathrm{ex}} t_{0}<0.5$, the system is in a low density (LD) phase. The stationary density profile $m^{\text {st }}(z)$ on the mRNA is governed by the entry rate $\omega_{\text {on }}$. The density profile is flat with an exponential tail near the exit boundary. The stationary bulk density value $m^{\text {st }}$ is determined by equating the entry flux $\omega_{\text {on }}\left(1-m^{\text {st }}\right)$ with the bulk current given by $m^{\text {st }}(1-$ $\left.m^{\text {st }}\right) / t_{0}$ and has a value $m^{\text {st }}=\omega_{\text {on }} t_{0}$. As a consequence, the average flux is $\omega_{\text {on }}\left(1-\omega_{\text {on }} t_{0}\right)$. 
Fig. 2 Phase diagram of the TASEP as a function of entry rate $\omega_{\text {on }}$ and exit rate $\omega_{\text {ex }}$. As mentioned before, these rates are related to the usual TASEP parameters $\alpha$ and $\beta$ via $\alpha=\min \left(\omega_{\mathrm{on}} t_{0}, 1\right)$ and $\beta=\min \left(\omega_{\mathrm{ex}} t_{0}, 1\right)$

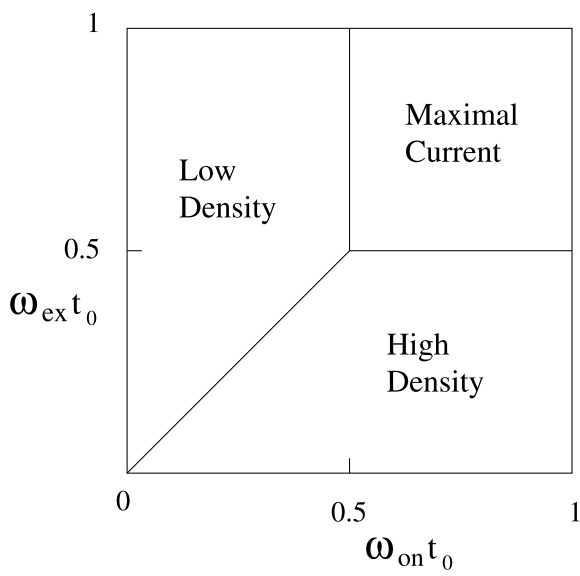

Steady state demands a constant flux throughout the mRNA, fixing the density $m^{\text {st }}$ at the exit site to the value $m^{\text {st }}(L)=\left(1-\omega_{\text {on }} t_{0}\right) \omega_{\text {on }} / \omega_{\text {ex }}$. Finally, disregarding the exponential tail near the exit boundary, the stationary number of ribosomes in this phase is given by $M_{\mathrm{LD}}^{\mathrm{st}}=\omega_{\mathrm{on}} t_{0} L$.

For $\omega_{\mathrm{ex}} t_{0}<\omega_{\mathrm{on}} t_{0}<0.5$, the entry rate exceeds the exit rate and the system goes into a high density (HD) phase. In this case, the exit boundary controls the average density. The stationary density profile $m^{\text {st }}(z)$ again is fixed by imposing that the exit current or flux $m^{\text {st }} \omega_{\text {ex }}$ must equal the bulk current $m^{\text {st }}\left(1-m^{\text {st }}\right) / t_{0}$. This leads to the bulk density value $m^{\text {st }}=1-\omega_{\mathrm{ex}} t_{0}$ and there is an exponential tail at the entry boundary with the density at the entry site being $m^{\text {st }}(1)=1-\left(1-\omega_{\mathrm{ex}} t_{0}\right) \omega_{\mathrm{ex}} / \omega_{\mathrm{on}}$. Finally, disregarding the exponential tail near the entry boundary, the stationary number of ribosomes in this phase is given by $M_{\mathrm{HD}}^{\mathrm{st}}=\left(1-\omega_{\mathrm{ex}} t_{0}\right) L$.

Increasing both $\omega_{\text {on }} t_{0}$ and $\omega_{\text {ex }} t_{0}$ beyond 0.5 leads to the maximal current (MC) phase. The ribosome flux here is fixed at the value $1 /\left(4 t_{0}\right)$, which is the maximum value possible in the steady state. It is independent of the entry and exit rates. This flux fixes the densities at the entry and exit ends: $m^{\text {st }}(1)=1-1 /\left(4 \omega_{\text {on }} t_{0}\right)$ and $m^{\text {st }}(L)=1 /\left(4 \omega_{\text {ex }} t_{0}\right)$. The density profile $m^{\text {st }}$ shows a power law decay at both ends towards the bulk density value of $m^{\text {st }}=1 / 2$. In the limit of large lattice size $L$, the stationary number of ribosomes in this phase is given by $M_{\mathrm{MC}}^{\mathrm{st}}=L / 2$.

Our results deal with the evolution towards the phases described above and we will see that the entry and exit rates not only determine the steady state phases but also affect the behavior in the time dependent regime.

\subsection{Results for $t<L t_{0}$}

The first ribosome entering the mRNA will take a time $L t_{0}$ to reach the other end since it moves forward by one unit at each time step. Thus for time $t<L t_{0}$, the effect of the exit boundary does not come into play. Therefore, any result in this regime will be independent of the exit rate $\omega_{\mathrm{ex}}$. In fact, one can argue that in this time regime, the behavior close to the entry end will always be as if the value of $\omega_{\mathrm{ex}} t_{0}$ is one. Assuming that correlations in the system are short ranged, the entry end cannot distinguish between a completely open exit boundary with $\omega_{\mathrm{ex}} t_{0}=1$ and no exit boundary at all since there is no restriction to the motion of ribosomes, except for their own hard core repulsion that is effective in both cases. 
Fig. 3 Number of ribosomes on the mRNA $M(t)$ versus time $t / t_{0}$. (a) Entry rate $\omega_{\text {on }}=0.3 / t_{0}$ and exit rate $\omega_{\mathrm{ex}}=0.1 / t_{0}$ and $0.9 / t_{0}$ (overlapping data). The straight line with slope 0.21 completely overlaps with the data showing excellent agreement. Thus for $\omega_{\text {on }} t_{0}<0.5$ and $t<L t_{0}$, we have

$M(t)=\omega_{\text {on }}\left(1-\omega_{\text {on }} t_{0}\right) t$. (b) Entry rate $\omega_{\mathrm{on}}=0.7 / t_{0}$ and exit rate $\omega_{\mathrm{ex}}=0.1 / t_{0}$ and $0.9 / t_{0}$ (overlapping data). The straight line has a slope 0.25 . The matching here is good but not as good as in the case with $\omega_{\text {on }} t_{0}<0.5$ case. The length of the mRNA strand is $L=1024$ and the data has been averaged over $10^{3}$ trajectories for both figures
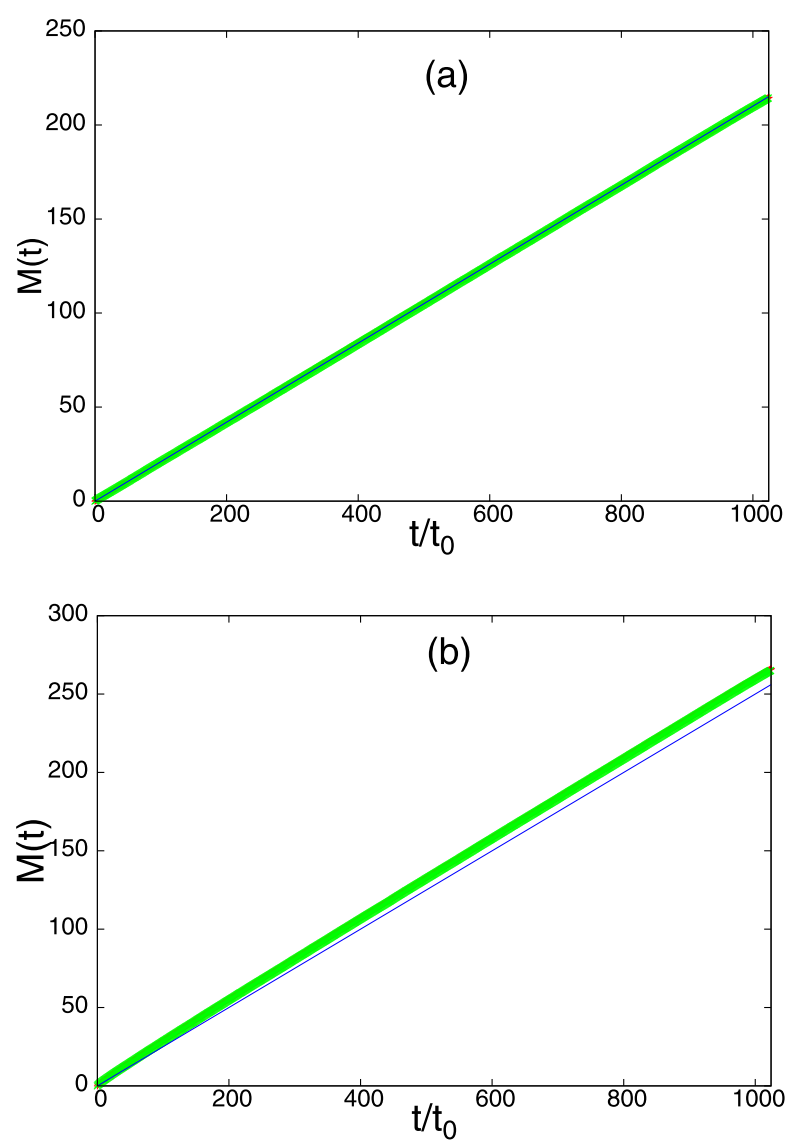

Therefore, guided by the steady state results, we predict that for times $t<L t_{0}$ the system shows two instead of three different kinds of behavior. Namely, for $\omega_{\text {on }} t_{0}<1 / 2$, the system behavior near the entry boundary is expected to be like that in a low density phase, implying that the entry flux of ribosomes should be $\omega_{\text {on }}\left(1-\omega_{\text {on }} t_{0}\right)$. For $\omega_{\text {on }} t_{0}>1 / 2$, instead, it is expected that the entry flux will be $1 / 4 t_{0}$ as in the maximal current phase.

Figure 3(a) shows the number of ribosomes on the mRNA, $M(t)$, as a function of $t / t_{0}$ for $\omega_{\text {on }} t_{0}=0.3$. According to our argument above, we expect the average ribosome number to behave as

$$
M(t)=\omega_{\mathrm{on}}\left(1-\omega_{\mathrm{on}} t_{0}\right) t \quad \text { for } t<L t_{0}
$$

and we see in Fig. 3(a) that this fits the data very well. Figure 3(b) shows the simulation data for $M(t)$ versus $t / t_{0}$ for $\omega_{\mathrm{on}} t_{0}=0.7$, which has been compared to the approximation, $M(t)=0.25 t / t_{0}$. We see that, interestingly, the entry flux of ribosomes here is slightly higher than the expected value of $1 / 4 t_{0}$, which, in fact, is the highest possible ribosome entry flux in the steady state. Why does our assumption of a steady state-like behavior near the entry boundary not work so well for $\omega_{\text {on }} t_{0}>0.5$ ?

A look at the density profiles $m(z, t)$ on the mRNA provides some qualitative understanding. For $\omega_{\mathrm{on}} t_{0}<0.5$, we see in Fig. 4(a) that there is a formation of a quasi-steady state 
Fig. 4 Density profile $m(z, t)$ as a function of the ribosome position $z$ for different choices of the time $t$. (a) Entry rate $\omega_{\text {on }}=0.3 / t_{0}$, exit rate $\omega_{\mathrm{ex}}=0.9 / t_{0}$ and mRNA length $L=1024$ in units of the lattice parameter $l_{0}$. The figure shows data for different times that increase from left to right, $t=100 t_{0}$ (yellow, leftmost), $t=300 t_{0}$ (light blue), $t=700 t_{0}$ (violet), $t=1100 t_{0}$ (blue), $t=1500 t_{0}$ (green) and $t=2500 t_{0}$ (red, rightmost). The straight line shows $m(z)=0.3$.

(b) Entry rate $\omega_{\text {on }}=0.8 / t_{0}$, exit rate $\omega_{\mathrm{ex}}=0.9 / t_{0}$ and $\mathrm{mRNA}$ size $L=1024$. The figure shows data for different times that increase from left to right, $t=100 t_{0}$ (yellow, leftmost), $t=200 t_{0}$ (light blue), $t=400 t_{0}$ (violet), $t=600 t_{0}$ (blue), $t=800 t_{0}$ (green) and $t=1000 t_{0}$ (red, rightmost). The data for both figures has been averaged over $10^{5}$ trajectories
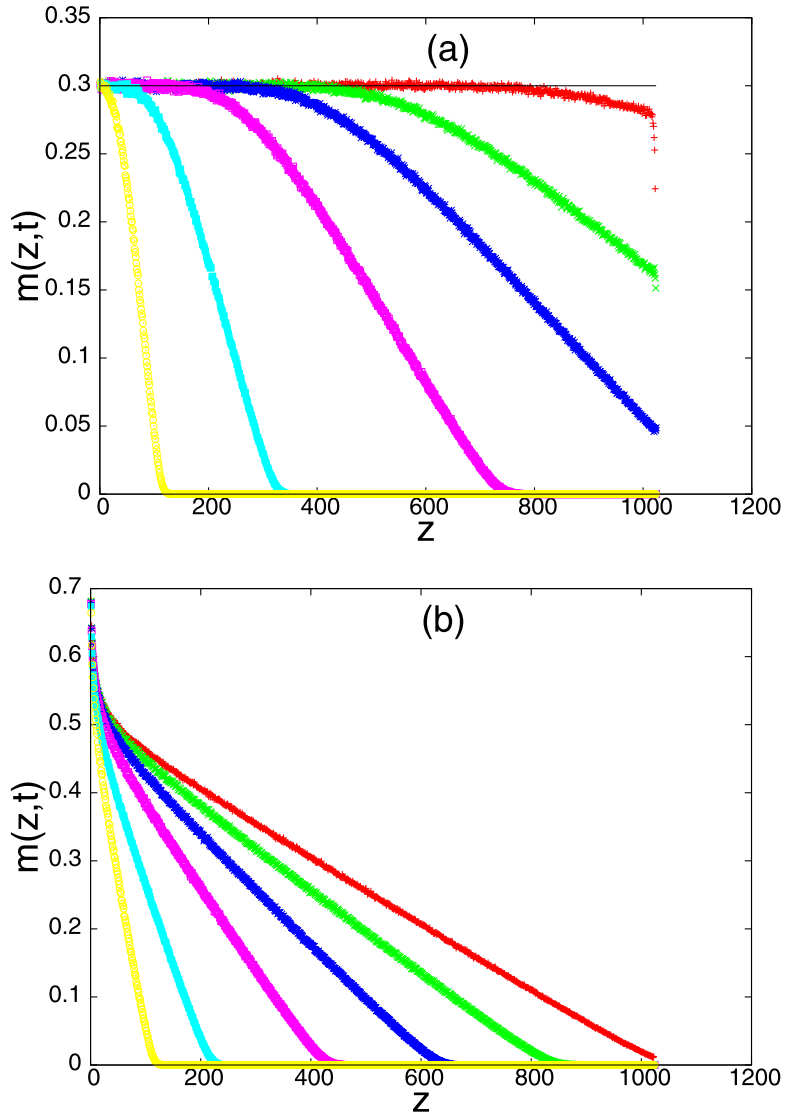

region of constant density — same as the steady state value of $\omega_{\text {on }} t_{0}$ - near the entry boundary, even at small times. This region leads to the entry flux value of $\omega_{\text {on }}\left(1-\omega_{\text {on }} t_{0}\right)$. On the other hand, for $\omega_{\text {on }} t_{0}>0.5$ as shown in Fig. 4(b), we do not see the emergence of such a constant density region, even at larger times. Our argument above assumes short range decay of correlations so that the entry boundary does not differentiate between a hindrancefree motion and a completely open boundary at the other end. We know that the steady state density correlation in the low density phase is exponentially decaying while in the maximal current phase, it is long ranged, showing an algebraic decay. This suggests that our assumption of short range decay of correlations might not hold in the $\omega_{\text {on }} t_{0}>0.5$ case, leading to a deviation from the value expected from the steady-state like behavior.

\subsection{Results for $t>L t_{0}$}

We now consider times larger than $L t_{0}$. The ribosomes start reaching the exit boundary, the exit rate $\omega_{\text {ex }}$ may now come into play, and the system will start approaching one of the three steady state phases in Fig. 2 depending on the values of $\omega_{\mathrm{on}}$ and $\omega_{\mathrm{ex}}$. 
Fig. 5 Number $M(t)$ of ribosomes on the mRNA versus time $t / t_{0}$. (a) LD case: Entry rate $\omega_{\text {on }}=0.3 / t_{0}$ and exit rate $\omega_{\mathrm{ex}}=0.9 / t_{0}$. The broken straight line with finite slope shows the behavior for $t<L t_{0}$ and the broken horizontal line shows the steady state ribosome number $M^{\text {st }}$. (b) HD case: Entry rate $\omega_{\mathrm{on}}=0.7 / t_{0}$ (upper data set) and $\omega_{\text {on }}=0.2 / t_{0}$ (lower data set); exit rate $\omega_{\mathrm{ex}}=0.1 / t_{0}$ for both. The broken straight lines have slopes $0.25-0.1(1-0.1)=0.16$ according to (8) and $0.2(1-0.2)-0.1(1-0.1)=0.07$ according to (7) and show a good fit to the intermediate regime with $t>L t_{0}$. (c) MC case: Entry rate $\omega_{\mathrm{on}}=0.7 / t_{0}$ and exit rate $\omega_{\mathrm{ex}}=1.0 / t_{0}$. The broken straight line with finite slope shows the behavior for $t<L t_{0}$ and the broken horizontal line shows the steady state ribosome number. In all three cases, the mRNA length $L=1024$ in units of the lattice parameter $l_{0}$ and the data has been averaged over $10^{3}$ trajectories
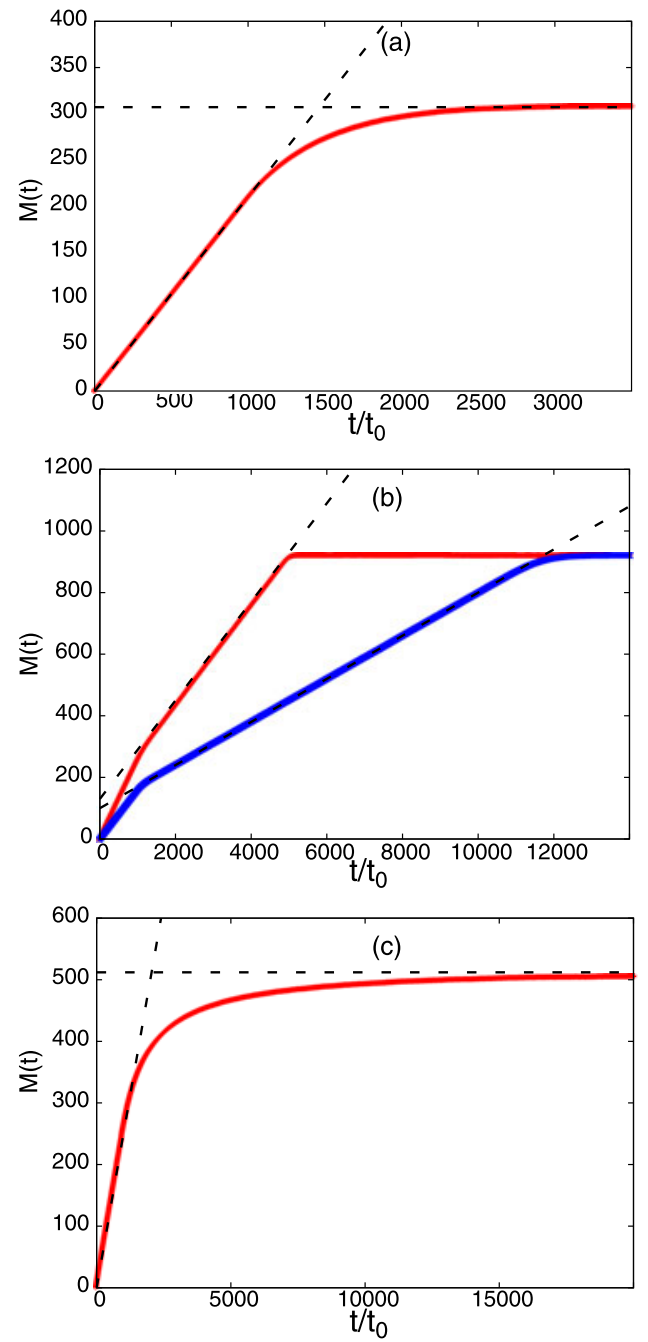

\subsubsection{Approach to Low Density Phase}

The steady state for $\omega_{\mathrm{on}} t_{0}<\omega_{\mathrm{ex}} t_{0}$ and $\omega_{\text {on }} t_{0}<0.5$ is a low density phase with the number of ribosomes in the steady state being $M^{\mathrm{st}}=\omega_{\mathrm{on}} L$. As we have seen in the previous section, the number of ribosomes loaded on the mRNA at time $t=L t_{0}$ will be $M\left(L t_{0}\right)=\omega_{\text {on }}(1-$ $\left.\omega_{\text {on }} t_{0}\right) L t_{0}$. Thus, a total of $M^{\text {st }}-M\left(L t_{0}\right)=\omega_{\text {on }}^{2} t_{0}^{2} L$ more ribosomes will be added in the $t>L t_{0}$ time range to reach steady state. As we see in Fig. 5(a), the long-time behavior for $t>L t_{0}$ interpolates smoothly between the two asymptotic regimes.

\subsubsection{Approach to High Density Phase}

The steady state for $\omega_{\mathrm{ex}} t_{0}<\omega_{\mathrm{on}} t_{0}, \omega_{\mathrm{ex}} t_{0}<0.5$ is a high density phase with the exit boundary controlling the density profile, which has the value $m^{\text {st }}=1-\omega_{\text {ex }} t_{0}$. If $\omega_{\text {on }} t_{0}<0.5$, then at times $t>L t_{0}$ but before the steady state is reached, we can make the assumption that the 
Fig. 6 Density profile $m(z, t)$ as a function of the ribosome position $z$ for various times. (a) HD case: Entry rate $\omega_{\text {on }}=0.2 / t_{0}$, exit rate $\omega_{\mathrm{ex}}=0.1 / t_{0}$. (b) HD case: Entry rate $\omega_{\text {on }}=0.7 / t_{0}$, exit rate $\omega_{\mathrm{ex}}=0.1 / t_{0}$. In both cases, the four data sets correspond to $t / t_{0}=400,800,2000$, and 4000 as indicated in the subfigures. Likewise, in both subfigures, the mRNA length $L=1024$ and the data has been averaged over $10^{5}$ trajectories
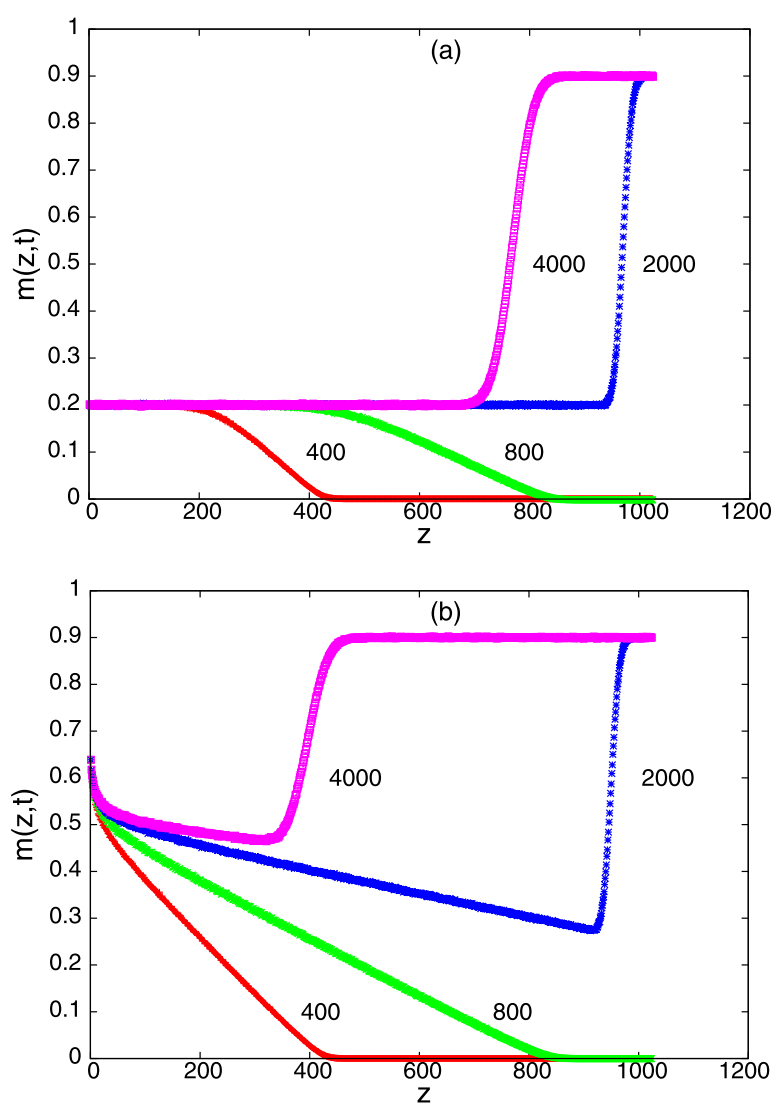

entry is controlled by $\omega_{\mathrm{on}}$, leading to ribosome entry flux of $\omega_{\mathrm{on}}\left(1-\omega_{\mathrm{on}} t_{0}\right)$ and the exit is controlled by $\omega_{\mathrm{ex}}$ leading to an exit per unit time of $\omega_{\mathrm{ex}}\left(1-\omega_{\mathrm{ex}} t_{0}\right)$. Thus the equation that governs the time evolution of the number of ribosomes at intermediate times is given by

$$
\frac{d M(t)}{d t}=\omega_{\mathrm{on}}\left(1-\omega_{\mathrm{on}} t_{0}\right)-\omega_{\mathrm{ex}}\left(1-\omega_{\mathrm{ex}} t_{0}\right)
$$

which leads to the lower broken line in Fig. 5(b). This reasoning is supported by the density profiles $m(z, t)$ on the mRNA at different times. We can clearly see in Fig. 6(a) that at larger times, there is a region with density equal to $\omega_{\text {on }} t_{0}$ near the entry boundary (left) and $1-\omega_{\mathrm{ex}} t_{0}$ near the exit boundary (right).

For $\omega_{\text {on }} t_{0}>0.5$ we can again make a similar approximation, with the value of the entry flux being $1 / 4 t_{0}$. As illustrated by the upper broken line in Fig. 5(b), the slope for $M(t)$ versus $t / t_{0}$ in the range $t>L t_{0}$ is well fitted by the formula

$$
\frac{d M(t)}{d t}=\frac{1}{4 t_{0}}-\omega_{\mathrm{ex}}\left(1-\omega_{\mathrm{ex}} t_{0}\right)
$$

The corresponding density profiles in Fig. 6(b) again show that entry and exit boundaries control the densities on the two adjacent mRNA segments. 


\subsubsection{Approach to Maximal Current Phase $\left(\omega_{\mathrm{on}} t_{0}>0.5, \omega_{\mathrm{ex}} t_{0}>0.5\right)$}

The steady state here is a maximal current phase. We see a nonlinear regime for $t>L t_{0}$ (Fig. 5(c)), again, we do not have a simple argument for the behavior in this regime.

\section{Effect of mRNA Degradation}

We now consider the effect of mRNA degradation on the process of translation. As we have mentioned in the introduction, the process of degradation leads to an ensemble of mRNA with a distribution of ages. The younger mRNA, where the translation has just begun, have ribosomes only near the entry boundary while the older ones, which have reached a steady state, have a more homogeneous ribosome density. The quantities recorded by the experiments represent an average over this ensemble of mRNA and this is what we want to capture in our model. Partially degraded mRNA may also contribute to this average in the experimental data but we assume that this effect is small, specially in prokaryotes, where the mRNA fragments are quickly digested. As mentioned in Sect. 2, the averages over mRNAs of different ages will be indicated with the symbols $\langle\cdot\rangle$. We have assumed a fixed degradation rate $\omega_{\mathrm{de}}$ leading to the mRNA age distribution $\phi_{A}$ given in (1). We have numerically calculated the quantities of interest, namely - the average number of ribosomes $\langle M\rangle$ as a function of mRNA coding region length $L$, the average density profile $m(z, t)$ of ribosomes as a function of position $z$ along the mRNA and the average probability distribution $\Pi_{n}$ of the number of ribosomes present on the mRNA. Our approach is to simulate the TASEP, starting with an empty mRNA, and then stop and take data at a random time chosen according to the age distribution $\phi_{A}$. This process is repeated for a large number of times and finally an average is taken over data from each configuration. We compare this data with approximations developed using results of the previous section.

The parameter values have been chosen as follows [16]: The velocity $v_{0}$ of unhindered ribosomes has been taken to be 60 footprints (fp) per minute; a footprint refers to the length $l_{0}$ of the ribosome, which is approximately 10 codons. For very low density and collisionless conditions, this would correspond to moving one footprint or one mRNA site per second by every ribosome. Thus one Monte-Carlo move, which corresponds to $t_{0}$ and involves a number of updates equal to the total number of ribosomes on the mRNA, represents one second. Assuming a typical life time of mRNA to be between 2 and 5 minutes, we take $\omega_{\mathrm{de}}=1 / 3.5 \mathrm{~min}^{-1}=0.00476 \mathrm{sec}^{-1}$. As we have seen in the previous section, the variation of the entry and exit rates not only leads to different steady states, but also to different behavior in the time dependent regime when the system is approaching steady state. We now discuss the effect of degradation in the different regions of parameter space.

\subsection{Low Density (LD) Case}

We consider $\omega_{\text {on }} t_{0}<\omega_{\text {ex }} t_{0}, \omega_{\text {on }} t_{0}<0.5$ where the steady state is a low density phase with stationary density profile $m^{\text {st }}=\omega_{\text {on }} t_{0}$. Our numerical results for the average density profile, $\langle m(z)\rangle$, as a function of the distance $z$ from the entry end, and the average density on the mRNA, $\langle M\rangle / L$, as a function of the total number of sites $L$ is shown in Figs. 7(a) and 8(a) respectively.

The numerical results have been compared to results from the following approximation. When the system is approaching the low density phase, we have seen in Fig. (5a) that the number of ribosomes rises linearly, $\langle M(t)\rangle=\omega_{\text {on }}\left(1-\omega_{\text {on }} t_{0}\right) t$, until time $t=L t_{0}$ and then the behavior changes to a nonlinear one until the steady state value $M^{\text {st }}=\omega_{\text {on }} t_{0} L$ is reached. We will neglect the nonlinear portion, which is relatively small, and assume that the number 
Fig. 7 Average density profile $\langle m\rangle$ as a function of ribosome position $z$. (a) LD case: Entry rate $\omega_{\text {on }}=0.2 / t_{0}$, exit rate $\omega_{\mathrm{ex}}=0.9 / t_{0}$, degradation rate $\omega_{\mathrm{de}}=0.00476 / t_{0}$ and different values of the mRNA length $L=60$ (red), 120 (green), 200 (blue). All data points for the different $L$-values lie on top of each other apart from those data points that correspond to $z$-values close to $z=L$. The analytical expression (11) leads to the solid line, which is hardly visible since it is in very good agreement with the data. (b) HD case: Entry rate $\omega_{\text {on }}=0.2 / t_{0}$, exit rate $\omega_{\mathrm{ex}}=0.1 / t_{0}$, degradation rate $\omega_{\mathrm{de}}=0.00476 / t_{0}$ and different values of $L$ : from left to right, the three data sets correspond to $L=60$ (red), 120 (green), 200 (blue). The solid lines are obtained from the analytical expression (18). (c) MC case: Entry rate $\omega_{\text {on }}=0.8 / t_{0}$, exit rate $\omega_{\mathrm{ex}}=0.9 / t_{0}$, degradation rate $\omega_{\mathrm{de}}=0.00476 / t_{0}$ and $L=60$ (red), 120 (green), 200 (blue). Again, all data points lie on top of each other apart from those data points that correspond to $z$-values close to $z=L$. The data for all three figures has been averaged over $10^{5}$ trajectories
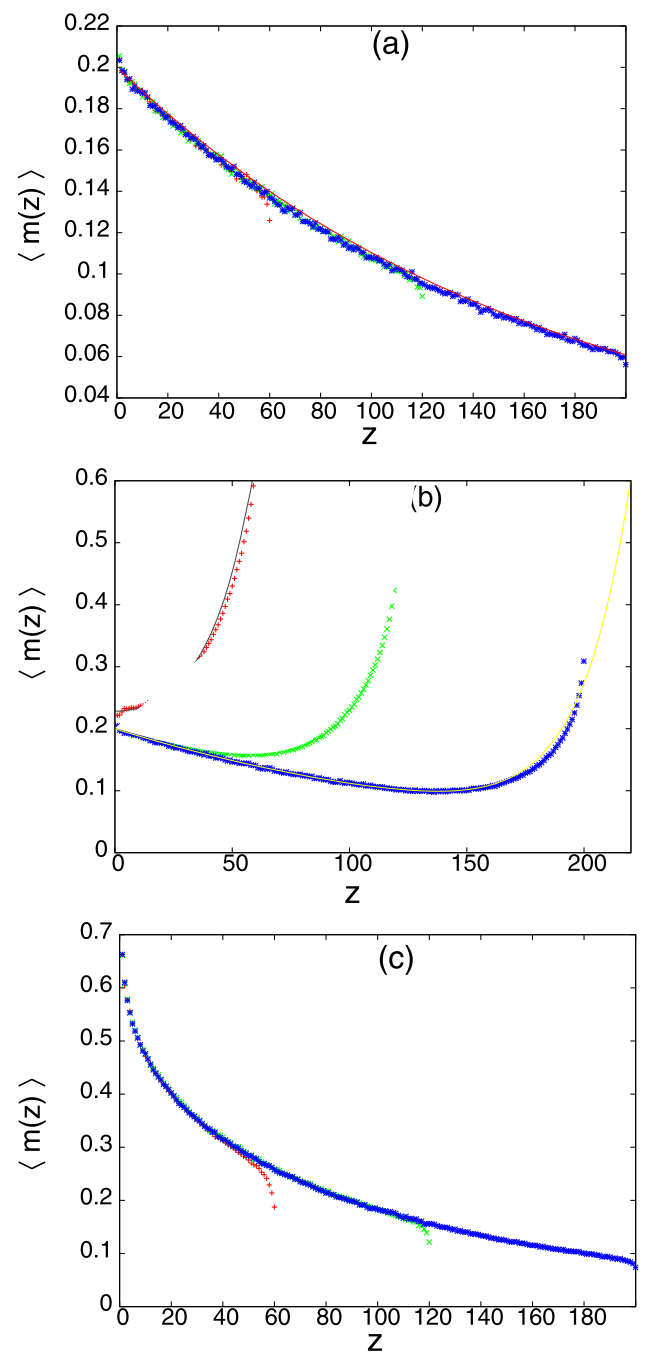

of ribosomes increases in a linear manner all the way to the steady state (see Fig. 9(a)). The density profile $m(z, t)$ is approximated as a front of uniform density $\omega_{\mathrm{on}} t_{0}$ that starts from the entry boundary and moves towards the exit boundary,

$$
m(z, t)= \begin{cases}0 & 0<t \leq \frac{z}{v}, \\ \omega_{\text {on }} t_{0} & t>\frac{z}{v},\end{cases}
$$

where the velocity of the front $v=v_{0}\left(1-\omega_{\text {on }} t_{0}\right)$, which ensures that the ribosome number $M(t)$ rises with the rate $\omega_{\text {on }}\left(1-\omega_{\text {on }} t_{0}\right)$. The time to reach steady state therefore will be $t_{s t}=L / v$ for $v$ expressed in footprints per second and we have

$$
M(t)= \begin{cases}\omega_{\mathrm{on}}\left(1-\omega_{\mathrm{on}} t_{0}\right) t & 0<t \leq L / v \\ \omega_{\mathrm{on}} t_{0} L & t>L / v\end{cases}
$$

where $L$ is the length of the mRNA in units of footprints. 
Fig. 8 Average density $\langle M\rangle / L$ versus mRNA length $L$. (a) LD case: Entry rate $\omega_{\text {on }}=0.2 / t_{0}$, exit rate $\omega_{\mathrm{ex}}=0.9 / t_{0}$ and degradation rate

$\omega_{\mathrm{de}}=0.00476 / t_{0}$. The simulation data are well described by the solid line obtained from the analytical expression (12). (b) HD case: Entry rate $\omega_{\text {on }}=0.2 / t_{0}$, exit rate $\omega_{\mathrm{ex}}=0.1 / t_{0}$ and degradation rate $\omega_{\mathrm{de}}=0.00476 / t_{0}$. The line corresponding to the prediction from (19) again shows good agreement with data points obtained from simulations.

(c) MC case: Entry rate $\omega_{\text {on }}=0.8 / t_{0}$, exit rate $\omega_{\mathrm{ex}}=0.9 / t_{0}$ and degradation rate $\omega_{\mathrm{de}}=0.00476 / t_{0}$. The points correspond to the data and the line provides a guide to the eye. In all three figures, each data point is a result of averaging over $10^{5}$ trajectories
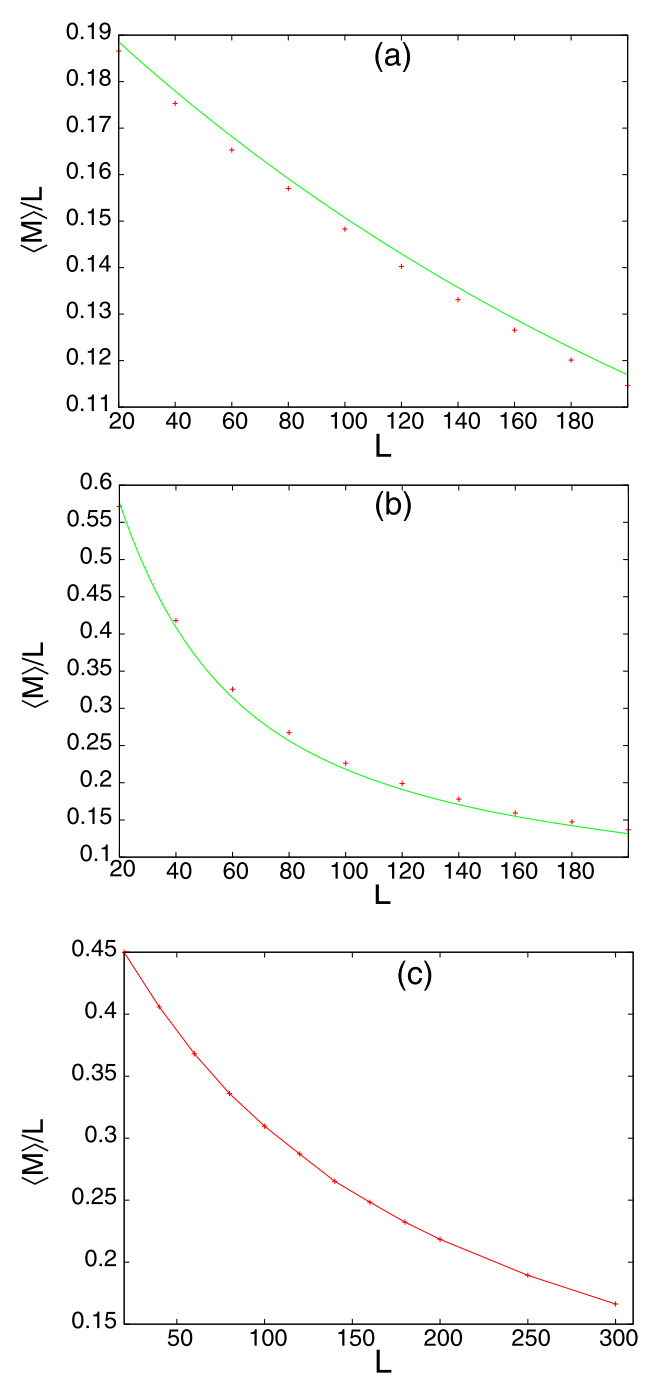

Using the above equations, we can calculate the average over the age distribution of the density profile from (5) as

$$
\langle m(z)\rangle=\omega_{\mathrm{on}} t_{0} \exp \left(-\frac{\omega_{\mathrm{de}} z}{v}\right)
$$

as well as the average density from (4) as

$$
\frac{\langle M\rangle}{L}=\frac{\omega_{\mathrm{on}}\left(1-\omega_{\mathrm{on}} t_{0}\right)}{\omega_{\mathrm{de}} L}\left[1-\exp \left(-\frac{\omega_{\mathrm{de}} L}{v}\right)\right] .
$$

The results from the above equations are compared with numerical data from our simulations and as we see in Figs. 7(a) and 8(a), the approximation works very well.

Another quantity that we have numerically computed is the average probability distribution, $\Pi_{n}$, of the total number of ribosomes, $n$ as defined in (2). A plot of this quantity for 
Fig. 9 Average number $M$ of ribosomes on the mRNA versus time $t / t_{0}$. (a) LD case: Entry rate $\omega_{\text {on }}=0.3 / t_{0}$ and exit rate $\omega_{\mathrm{ex}}=0.9 / t_{0}$. Data from simulations is shown in red and the broken line shows the approximation as in (10). (b) HD case: Entry rate $\omega_{\mathrm{on}}=0.2 / t_{0}$ and exit rate $\omega_{\mathrm{ex}}=0.1 / t_{0}$. Data from simulations is shown in red and the broken line shows the time evolution as in (17), which provides a very good approximation to the data. For both figures, $L=1024$ and the data has been averaged over $10^{3}$ trajectories
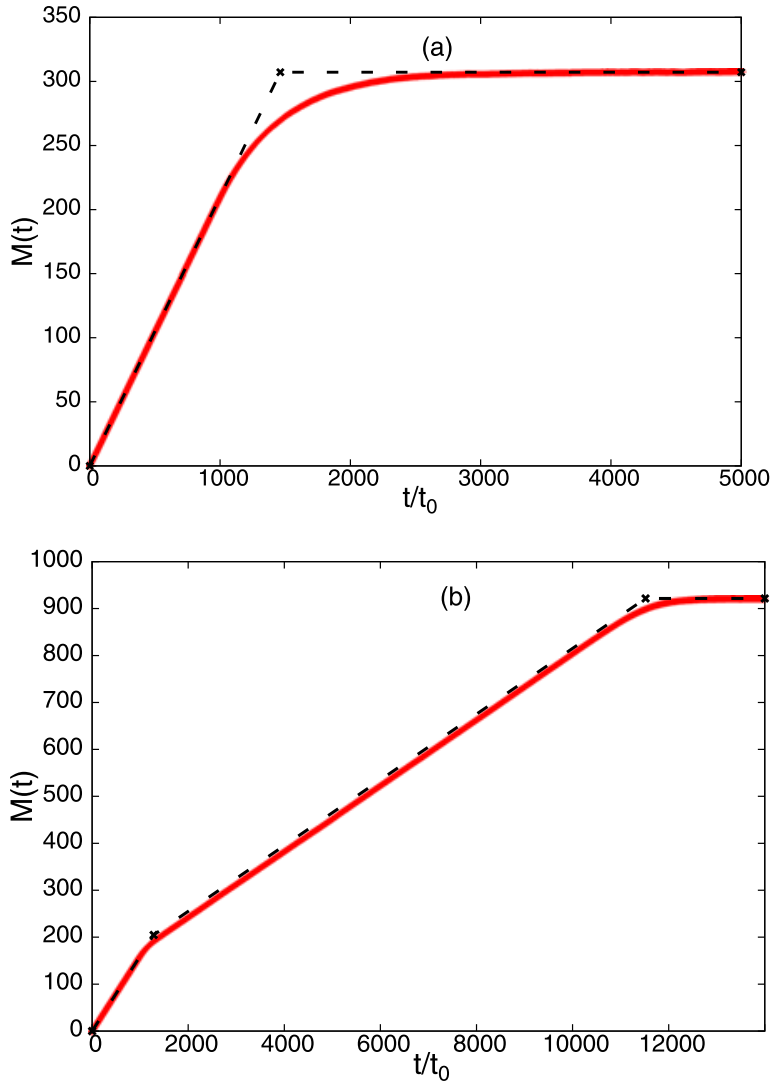

several choices of mRNA length $L$ is shown in Fig. 10(a). This quantity can experimentally be measured from ultracentrifugation experiments. For small mRNA lengths, the data from the model is close to the steady state data from a TASEP without degradation. This is to be expected since for very small mRNA, the degradation time can be long enough for the system to reach close to the steady state. For larger $L$, we see a distinct difference between the prediction from the model and the TASEP steady state behavior. We see a clear signature of the degradation in an additional peak at small values of $n$ which is different from the usual steady state distribution without degradation.

\subsection{High Density (HD) Case}

When $\omega_{\text {ex }} t_{0}<\omega_{\text {on }} t_{0}, \omega_{\text {ex }} t_{0}<0.5$, the exit boundary controls the steady state density and the system is in a high density phase. As we have seen before, even within this parameter regime, there is a difference in the approach towards steady state when $\omega_{\text {on }} t_{0}<0.5$ and $\omega_{\text {on }} t_{0}>0.5$ (Figs. 4 and 6). When $\omega_{\text {on }} t_{0}<0.5$, guided by Fig. 6(a), we can approximate the time dependent density profile as follows. Initially, a front of density $\omega_{\text {on }} t_{0}$ moves from the entry end to the exit end with speed $v_{1}=v_{0}\left(1-\omega_{\text {on }} t_{0}\right)$, like in the low density phase, until this front hits the exit boundary at time $t_{L}=L / v_{1}$. Then a front of density $\left(1-\omega_{\mathrm{ex}} t_{0}\right)$ starts going from the exit boundary towards the entry boundary with speed $v_{2}$ where this velocity 
Fig. 10 Probability distribution $\Pi_{n}$ of the number of ribosomes on the mRNA. (a) LD case: Entry rate $\omega_{\text {on }}=0.2 / t_{0}$, exit rate $\omega_{\mathrm{ex}}=0.9 / t_{0}$ and degradation rate $\omega_{\mathrm{de}}=0.00476 / t_{0}$ for three values of $L, 60$ (red), 120 (dark blue) and 200 (light blue). Also shown is data for the corresponding steady state distribution with no degradation for $L=60$ (green), 120 (violet) and 200 (yellow). (b) HD case: Entry rate $\omega_{\text {on }}=0.2 / t_{0}$, exit rate $\omega_{\mathrm{ex}}=0.1 / t_{0}$ and degradation rate $\omega_{\mathrm{de}}=0.00476 / t_{0}$ for three values of $L, 60$ (red), 120 (dark blue) and 200 (light blue). Also shown is data for the corresponding steady state distribution with no degradation for $L=60$ (green), 120 (violet) and 200 (yellow). (c) MC case: Entry rate $\omega_{\text {on }}=0.8 / t_{0}$, exit rate $\omega_{\mathrm{ex}}=0.9 / t_{0}$ and degradation rate $\omega_{\mathrm{de}}=0.00476 / t_{0}$ for three values of mRNA length $L, 60$ (red), 120 (dark blue) and 200 (light blue). Also shown is data for the corresponding steady state distribution without degradation.

Here again we have $L=60$ (green), 120 (violet) and 200 (yellow). The points in all the figures correspond to the data and the lines provide a guide to the eye. The data for all three figures has been averaged over $10^{6}$ trajectories. In all three cases, degradation leads to a broadening of the distributions and to a shift of the maxima towards smaller values of $n$
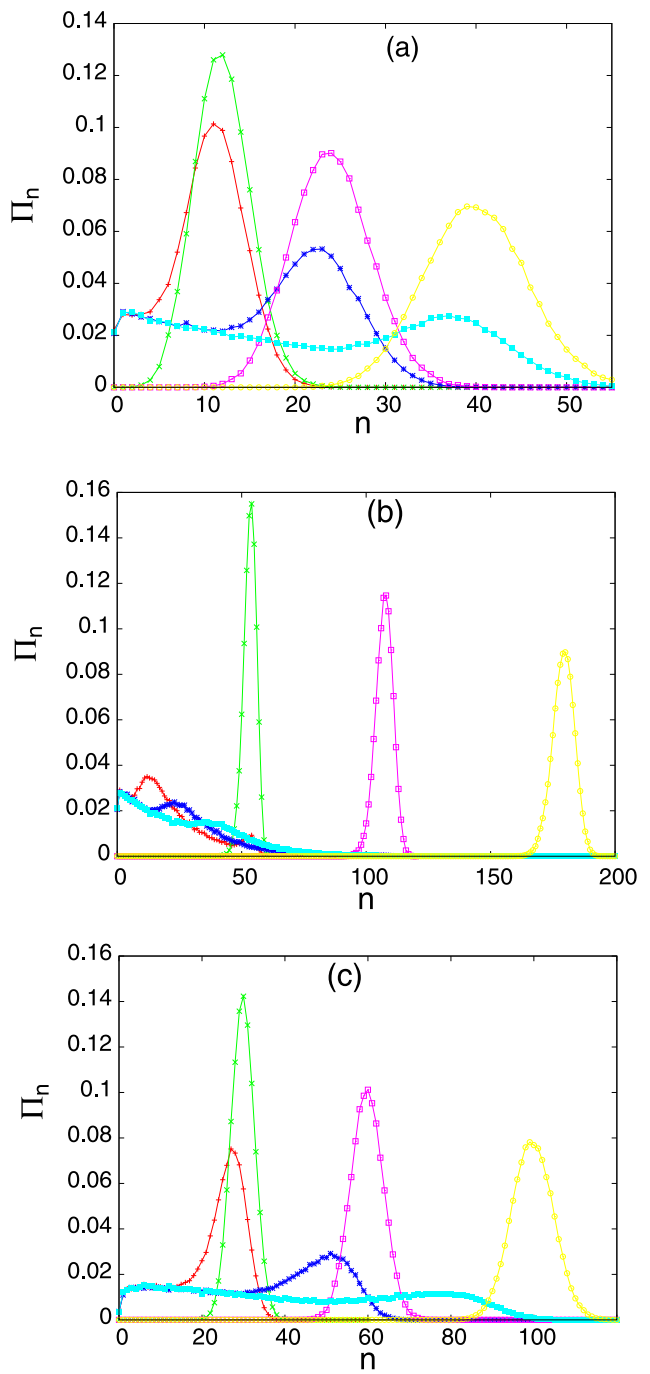

can be obtained by equating

$$
\frac{\mathrm{d} M(t)}{\mathrm{d} t}=\left(1-\omega_{\mathrm{ex}} t_{0}\right) v_{2}-\omega_{\mathrm{on}} t_{0} v_{2},
$$

to (7). This finally leads to

$$
v_{2}=\frac{v_{0}\left[\omega_{\mathrm{on}} t_{0}\left(1-\omega_{\mathrm{on}} t_{0}\right)-\omega_{\mathrm{ex}} t_{0}\left(1-\omega_{\mathrm{ex}} t_{0}\right)\right]}{1-\omega_{\mathrm{ex}} t_{0}-\omega_{\mathrm{on}} t_{0}} .
$$

Steady state is reached when this density front reaches the entry boundary at time $t_{s}$ determined by

$$
t_{s}=\frac{L}{v_{1}}+\frac{L}{v_{2}}
$$


Thus we can write

$$
m(z, t)= \begin{cases}0 & 0<t \leq \frac{z}{v_{1}}, \\ \omega_{\mathrm{on}} t_{0} & \frac{z}{v_{1}}<t \leq \frac{L}{v_{1}}+\frac{(L-z)}{v_{2}}, \\ 1-\omega_{\mathrm{ex}} t_{0} & t>\frac{L}{v_{1}}+\frac{(L-z)}{v_{2}} .\end{cases}
$$

The ribosome number is approximated by two piece-wise linear portions with different slopes (see Fig. 9(b)) and we can write

$$
M(t)= \begin{cases}\omega_{\mathrm{on}}\left(1-\omega_{\mathrm{on}} t_{0}\right) t & 0<t<t_{L}, \\ \omega_{\mathrm{on}}\left(1-\omega_{\mathrm{on}} t_{0}\right) t_{L}+\left[\omega_{\mathrm{on}}\left(1-\omega_{\mathrm{on}} t_{0}\right)-\omega_{\mathrm{ex}}\left(1-\omega_{\mathrm{ex}} t_{0}\right)\right]\left(t-t_{L}\right) & t_{L}<t<t_{s}, \\ \left(1-\omega_{\mathrm{ex}} t_{0}\right) L & t>t_{s}\end{cases}
$$

where $t_{s}$ is defined in (15) and $t_{L}=L / v_{1}$. The averaging over age distribution according to (5) and (4) then leads to

$$
\begin{aligned}
\langle m(z)\rangle= & \omega_{\mathrm{on}} t_{0} \exp \left(-\omega_{\mathrm{de}} \frac{z}{v_{1}}\right) \\
& +\left(1-\omega_{\mathrm{on}} t_{0}-\omega_{\mathrm{ex}} t_{0}\right) \exp \left(-\omega_{\mathrm{de}}\left(\frac{L}{v_{1}}+\frac{(L-z)}{v_{2}}\right)\right)
\end{aligned}
$$

and

$$
\begin{aligned}
\frac{\langle M\rangle}{L}= & \frac{\omega_{\mathrm{on}}\left(1-\omega_{\mathrm{on}} t_{0}\right)}{L \omega_{\mathrm{de}}}\left(1-\exp \left(-\omega_{\mathrm{de}} t_{L}\right)\right) \\
& +\frac{\omega_{\mathrm{on}}\left(1-\omega_{\mathrm{on}} t_{0}\right)-\omega_{\mathrm{ex}}\left(1-\omega_{\mathrm{ex}} t_{0}\right)}{L \omega_{\mathrm{de}}}\left(\exp \left(-\omega_{\mathrm{de}} t_{L}\right)-\exp \left(-\omega_{\mathrm{de}} t_{s}\right)\right) .
\end{aligned}
$$

Figures 7(b) and 8(b) show the comparison of the numerical data with prediction from the above formulas and we see that they work quite well. We see a distinct feature in the averaged density profile $\langle m(z)\rangle$ in Fig. 7(b) - a peak near the exit boundary. As we have seen in Fig. 4(a), after the ribosomes start reaching the exit boundary, a jam starts building up, which we have described above as a density front moving towards the entry boundary. This density front contributes more to the density near the exit boundary and leads to the peak in the average profile.

When $\omega_{\text {on }} t_{0}>0.5$, we have seen that the density does not evolve like a constant value front (Fig. 4(b)) and we can not extend the analysis above. Figure 11(a) shows the average density $\langle M\rangle / L$ as a function of mRNA size and the behavior is qualitatively similar to the $\omega_{\text {on }} t_{0}<0.5$ case. The averaged density profile $\langle m(z)\rangle$ in Fig. 11(b) again, similar to the case $\omega_{\text {on }} t_{0}<0.5$, shows a peak near the exit boundary arising from a backward moving density front.

We have also measured the probability distribution $\Pi_{n}$ for the HD case as shown in Fig. 10(b). The age-averaged distribution $\Pi_{n}$ for the system with degradation is much broader and shifted towards smaller value of $n$ compared to the distribution for the steady state without degradation. This change in behavior can be attributed to the larger time required to reach steady state. While the ribosomes start to reach the end codon at time of the order of $L t_{0}$, the time to reach steady state is still of the order of $L t_{0}$. Thus, if the mRNA-ribosome system works in the HD phase, we will find pronounced age effects in the ultracentrifugation data. 
Fig. 11 (a) HD case: Average density $\langle M / L\rangle$ versus $L$ for entry rate $\omega_{\text {on }}=0.7 / t_{0}$, exit rate $\omega_{\mathrm{ex}}=0.1 / t_{0}$ and degradation rate $\omega_{\mathrm{de}}=0.00476 / t_{0}$. The points show simulation results and the line provides a guide to the eye. (b) HD case: Average density profile $\langle m\rangle$ versus $z$ for entry rate $\omega_{\text {on }}=0.7 / t_{0}$, exit rate $\omega_{\mathrm{ex}}=0.1 / t_{0}$, degradation rate $\omega_{\mathrm{de}}=0.00476 / t_{0}$ and different values of the mRNA lenght $L$ : from left to right, the three data sets correspond to $L=60$ (red), 120 (green), 200 (blue). The data for both figures is a result of averaging over $10^{5}$ trajectories
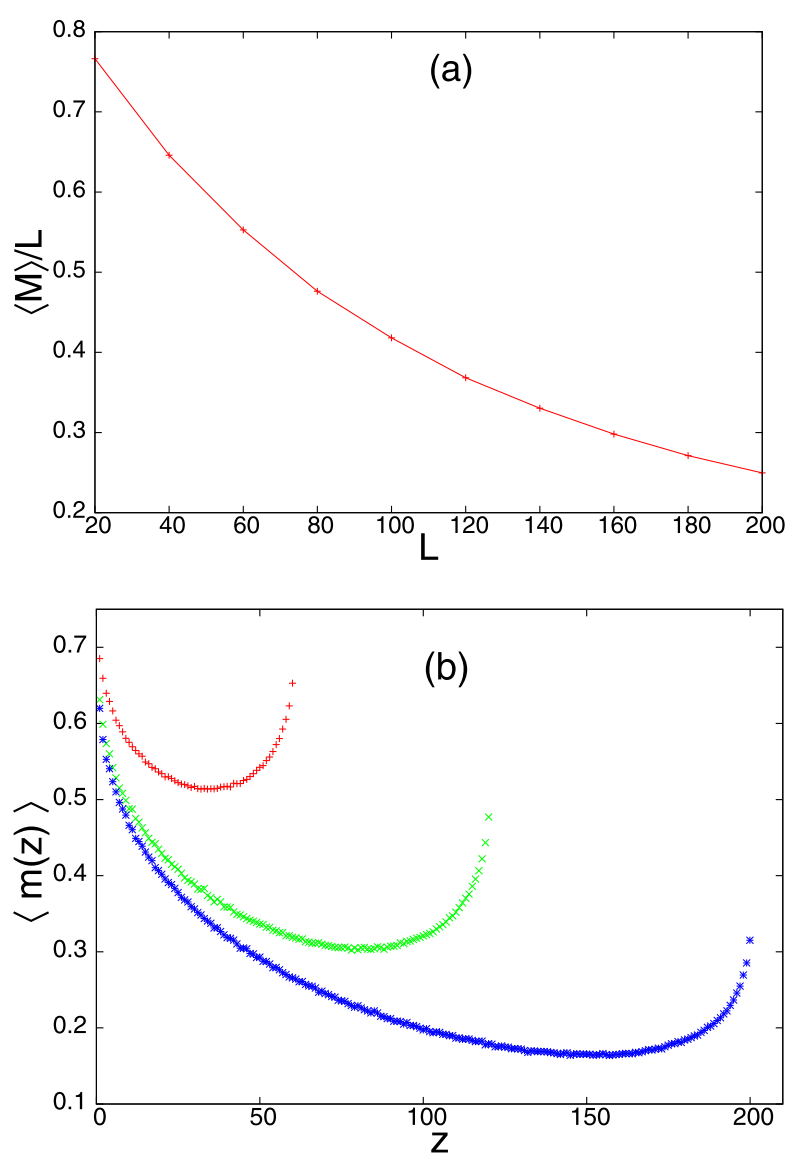

\subsection{Maximal Current (MC) Case}

When $\omega_{\mathrm{on}} t_{0}, \omega_{\mathrm{ex}} t_{0}>0.5$, the steady state of the system is a maximal current phase with a fixed density of half, independent of the entry and exit rates. The numerical results for the average density $\langle M\rangle / L$ as a function of mRNA length is shown in Fig. 8(c) and the average density profile $\langle m(z)\rangle$ versus $z$, expressed in footprints units has been shown in Fig. 7(c).

These results are qualitatively similar to those in the low density case. In the previous section, we had seen that the initial increase in number of ribosomes for $\omega_{\mathrm{on}} t_{0}>0.5$ is linear, with a coefficient larger than the $1 / 4 t_{0}$ expected from a simple argument. We had also seen that the density profile (Fig. 4(b)) does not have an expanding region of constant density as in the case $\omega_{\mathrm{on}} t_{0}<0.5$. Thus we cannot extend the analysis that we had used in the LD case to develop an analytic approximation.

Figure $10(\mathrm{c})$ shows the probability distribution, $\Pi_{n}$, of the number of ribosomes number $n$. We again see a significant difference from the steady state without decay and, as expected, the difference increases with increasing mRNA length. 


\section{Conclusions}

In this paper, we have studied the effect of degradation on the process of translation. We have taken the totally asymmetric simple exclusion process to describe ribosome movement on mRNA. The degradation has been introduced via an age distribution for the mRNA and the average over this distribution provides experimentally observable quantities. This averaging includes mRNAs of all ages-from very young, unloaded ones to older ones which have reached a steady state ribosome density. Therefore, before considering the effect of degradation, it is important to study the time evolution of a TASEP towards steady state from an initial condition of empty mRNA. Therefore, we have presented our results in two sections.

In Sect. 3, we have discussed the time evolution of the ribosome or particle density for the various parameter regions. We have seen that our knowledge of the steady state behavior of the system can provide an intuitive understanding of the initial time-dependent behavior. We have, in fact, used this understanding to develop a simple approximation that describes our numerical results quite well. The initial rise in the number of ribosomes is always linear in time with coefficients depending on the entry rate $\omega_{\text {on }}$, see Fig. 3. The ribosome entry flux for $\omega_{\text {on }} t_{0}>0.5$ has a value larger than $1 / 4 t_{0}$, see Fig. 3(b), which is an unexpected and interesting result since the entry flux value can never exceed $1 / 4 t_{0}$ in steady state conditions. For times larger than $L t_{0}$, the exit boundary becomes important, and for the high density phase parameters as given by $\omega_{\mathrm{ex}}<\omega_{\mathrm{on}}$ and $\omega_{\mathrm{ex}} t_{0}<0.5$, the entry and exit boundaries determine the fluxes in their vicinity, see Figs. 6(a) and (b), leading to a ribosome entry flux of $\omega_{\mathrm{on}}\left(1-\omega_{\mathrm{on}} t_{0}\right)$ and an exit flux $\omega_{\mathrm{ex}}\left(1-\omega_{\mathrm{ex}} t_{0}\right)$. These results for the time evolution of a TASEP are interesting in themselves and, as far as we know, new.

Section 4 addresses the biology problem at hand-the effect of degradation on protein translation. We have focused here on three experimentally measurable quantities: (i) the average density profile $\langle m(z)\rangle$ as a function of distance along the mRNA, (ii) the average probability distribution $\Pi_{n}$ of the number of ribosomes on the mRNA and (iii) the average number of ribosomes $\langle M\rangle$ on the mRNA. The initial phase of mRNA loading, before the system has reached its steady state, contributes significantly to the average over the age distribution. As a result, we find that there is a decrease in the averaged density profile $\langle m(z)\rangle$ as one moves away from the entry boundary, see (11) and Fig. 7(a). For parameters corresponding to the low density steady state, the profile is similar to the one in the simple model studied in [16]. We also reveal a new feature for the high density phase-a peak in the average density near the exit boundary, see (18) and Figs. 7(b) and 11(b). This peak can be understood in terms of a traffic jam caused by a low exit rate which contributes to the high density near the exit end.

As far as translation by ribosomes is concerned, we have focused on the effect of degradation, neglecting features such as ribosome drop off, effects of tRNA concentration etc. Our results show that small degradation times can lead to significant effects on polysome statistics. These results are relevant for prokaryotic organisms such as E. coli where the degradation mechanism is simple and the average age of the mRNA is comparable to the time scale of translation, both of which can be of the order of a few minutes [15, 25-27]. In prokaryotes, mRNA translation begins before the completion of transcription. In our study, we have implicitly assumed that the average translation rate is smaller than the average transcription rate. For $E$. coli, the average transcription rate was found to be 62 nucleotides/s or about 21 codons/s [28] whereas the average translation rate is estimated to be 600 codons/min or 10 codons/s [16]. Thus for this latter organism, the translation rate is indeed smaller than the transcription rate, and we may then ignore possible effects of co-transcriptional translation on the polysome statistics. Some results on the density profile [23] and probability of 
polysome size [20] are also available for eukaryotes such as the yeast $S$. cerevisiae, and it is expected that the translation profiles for other organisms will be explored using similar experimental methods. It will be interesting to see to what extent future experiments confirm our predictions. A comparison with the experimental data may require more detailed models which include other important features such as the effect of extended particles [29] and the sequence of codons $[23,30]$.

Acknowledgements We thank Sophia Rudorf, Carlus Deneke, and Stefan Klumpp for stimulating interactions. The research of A.N. was supported by the German Federal Ministry of Education and Research in the framework of GoFORSys. Figure 1 was designed in collaboration with Susann Valleriani.

Open Access This article is distributed under the terms of the Creative Commons Attribution Noncommercial License which permits any noncommercial use, distribution, and reproduction in any medium, provided the original author(s) and source are credited.

\section{References}

1. MacDonald, C.T., Gibbs, J.H., Pipkin, A.C.: Kinetics of biopolymerization on nucleic acid templates. Biopolymers 6, 1 (1968)

2. Shaw, L.B., Zia, R.K.P., Lee, K.H.: Totally asymmetric exclusion process with extended objects: a model for protein synthesis. Phys. Rev. E 68, 02190-1 (2003)

3. Dong, J.J., Schmittmann, B., Zia, R.K.P.: Inhomogeneous exclusion processes with extended objects: the effect of defect locations. Phys. Rev. E 76, 051113 (2007)

4. Romano, C.M., Thiel, M., Stansfield, I., Grebogi, C.: Queueing phase transition: theory of translation. Phys. Rev. Lett. 102, 198104 (2009)

5. Chou, T.: Ribosome recycling, diffusion, and mRNA loop formation in translational regulation. Biophys. J. 85, 755 (2003)

6. Derrida, B., Evans, M.R., Hakim, V., Pasquier, V.: Exact solution of a 1D asymmetric exclusion model using a matrix formulation. J. Phys. A 26, 1493 (1993)

7. Schuetz, G., Domany, E.: Phase transitions in an exactly soluble one-dimensional exclusion process. J. Stat. Phys. 72, 277 (1993)

8. Houseley, J., Tollervey, D.: The many pathways of RNA degradation. Cell 136, 763 (2009)

9. Kushner, S.R.: mRNA decay in prokaryotes and eukaryotes: different approaches to a similar problem. IUBMB Life 56, 585 (2004)

10. Deutscher, M.P.: Maturation and degradation of ribosomal RNA in bacteria. Prog. Mol. Biol. Transl. Sci. 85, 369 (2009)

11. Kaberdin, V.R., Blaesi, U.: Translation initiation and the fate of bacterial mRNAs. FEMS Microbiol. Rev. 30, 967 (2006)

12. Belasco, J.G.: All things must pass: contrasts and commonalities in eukaryotic and bacterial mRNA decay. Nat. Rev. Mol. Cell Biol. 11, 467 (2010)

13. Condon, C.: Maturation and degradation of RNA in bacteria. Curr. Opin. Microbiol. 10, 271 (2007)

14. Hu, W., Sweet, T.J., Chamnongpol, S., Baker, K.E. Coller, J.: Co-translational mRNA decay in Saccharomyces cerevisiae. Nature (London) 461, 225 (2009)

15. Mitarai, N., Sneppen, K., Pederson, S.: Ribosome collisions and translation efficiency: optimization by codon usage and mRNA destabilization. J. Mol. Biol. 382, 236 (2008)

16. Valleriani, A., Ignatova, Z., Nagar, A., Lipowsky, R.: Turnover of messenger RNA: polysome statistics beyond the steady state. Europhys. Lett. 89, 58003 (2010)

17. Valleriani, A., Zhang, G., Nagar, A., Ignatova, Z., Lipowsky, R.: Length-dependent translation of messenger RNA by ribosomes. Phys. Rev. E 83, 042903 (2011)

18. de Gier, J., Essler, F.H.L.: Exact spectral gaps of the asymmetric exclusion process with open boundaries. J. Stat. Mech. 2006, P12011 (2006)

19. Dudzinski, M., Schuetz, G.M.: Relaxation spectrum of the asymmetric exclusion process with open boundaries. J. Phys. A 33, 8351 (2000)

20. Arava, Y., Wang, Y., Storey, J.D., Jong, C.L., Brown, P.O., Herschlag, D.: Genome-wide analysis of mRNA translation profiles in Saccharomyces cerevisiae. Proc. Natl. Acad. Sci. USA 100, 3889 (2003)

21. Qin, X., Ahn, S., Speed, T.P., Rubin, G.M.: Global analyses of mRNA translational control during early Drosophila embryogenesis. Genome Biol. 8, R63 (2007) 
22. Eldad, N., Arava, Y.: A ribosomal density-mapping procedure to explore ribosome positions along translating mRNAs. In: Wilusz, J. (ed.) Methods in Molecular Biology. Post-Transcriptional Gene Regulation, vol. 419, p. 231. Humana Press, Totowa (2007)

23. Ingolia, N.T., Ghaemmaghami, S., Newman, J.R.S., Weissman, J.S.: Genome-wide analysis in vivo of translation with nucleotide resolution using ribosome profiling. Science 324, 218 (2009)

24. Morris, D.R.: Ribosomal footprints on a transcriptome landscape. Genome Biol. 10, 215 (2009)

25. Grunberg-Manago, M.: Messenger RNA stability and its role in control of gene expression in bacteria and phages. Annu. Rev. Genet. 33, 193 (1999)

26. Johannson, M., Lovmar, M., Ehrenberg, M.: Rate and accuracy of bacterial protein synthesis revisited. Curr. Opin. Microbiol. 11, 141 (2008)

27. Bernstein, J.A., Khodursky, A.B., Lin, P.-H., Lin-Chao, S. Cohen, S.N.: Global analysis of mRNA decay and abundance in Escherichia coli at single-gene resolution using two-color fluorescent DNA microarrays. Proc. Natl. Acad. Sci. USA 99, 9697 (2002)

28. Epshtein, V., Nudler, E.: Cooperation between RNA polymerase molecules in transcription elongation. Science 300, 801 (2003)

29. Lakatos, G., Chou, T.: Totally asymmetric exclusion processes with particles of arbitrary size. J. Phys. A, Math. Gen. 36, 2027 (2003)

30. Tuller, T., Carmi, A., et al.: An evolutionarily conserved mechanism for controlling the efficiency of protein translation. Cell 141, 344 (2010) 